\title{
Chronic Low Dose Organic Arsenic Induced Liver Structural Damage
}

Shahida $S^{a}$, Nor Zamzila $A^{b}$, Norlelawati $A T^{b}$, Jamalludin $A R^{c}, A z l i a n a A F^{b}$, Zunariah $B^{a}$

${ }^{a}$ Department of Basic Medical Sciences, Kulliyyah of Medicine, International Islamic University Malaysia.

bepartment of Pathology and Laboratory Medicine, Kulliyyah of Medicine, International Islamic University Malaysia.

'Department of Community Medicine, Kulliyyah of Medicine, International Islamic University Malaysia.

\section{ABSTRACT}

Introduction: Over the decades, organic arsenic has been thought to be less toxic than inorganic arsenic. Monosodium methylarsonate (MSMA) is a potent organoarsenical herbicide that is still being used in most Asian countries. Reported studies on the effects of organic arsenic are mainly to the gastrointestinal system, however there are limited research on its impacts to the liver. Therefore, this study aimed to investigate the effect of MSMA exposure on hepatocytes and liver sinusoidal endothelial cells (LSEC). Materials and Methods: Fourteen Sprague Dawley rats $(n=14)$ were divided equally into arsenic-exposed $(n=7)$ and control $(n=7)$ groups. The rats in arsenic-exposed group were given MSMA at $63.20 \mathrm{mg} / \mathrm{kg}$ daily for 6 months through oral gavage. While the rats in control group were given distilled water ad libitum. At the end of the duration, they were euthanized and underwent liver perfusion for tissue preservation. Liver tissues were harvested and processed for light microscopy, scanning and transmission electron microscopy. The findings were analysed descriptively. Results: MSMA had caused necrotic and apoptotic changes to the liver. Normal organelles morphology were loss in the hepatocytes while LSEC revealed defenestration. Conclusion: In this study, chronic low dose organic arsenic exposure showed evidence of toxicity to hepatocytes. Interestingly, LSEC demonstrated capillarization changes.

KEYWORDS: Low dose, chronic exposure, organic arsenic, liver

\section{INTRODUCTION}

Over the decades, organic arsenic has been thought to be less toxic than inorganic arsenic. Increasing evidences are pointing out that it may not be so. ${ }^{1-3}$ Monosodium methylarsonate (MSMA) is a potent organoarsenical herbicide that is still being used particularly in most Asian countries. It is an organic form of arsenic used as herbicides and pesticides for controlling weeds and insects. ${ }^{4-6}$ Majority of arsenic studies involved inorganic arsenic compounds as organic arsenic has been thought to be less toxic. ${ }^{7,8}$ Even though both inorganic and organic leave the

\section{Corresponding author:}

Asst. Prof. Dr. Zunariah Buyong

Department of Basic Medical Sciences,

Kulliyyah of Medicine,

International Islamic University Malaysia,

Jalan Sultan Ahmad Shah,

25200 Kuantan, Pahang, Malaysia.

Tel. No: +6095704500

Email : drzuna@iium.edu.my body through urine after several days and some even longer, ${ }^{9,10}$ majority of the latter is expelled more rapidly. ${ }^{11}$ Many experimental studies have shown the carcinogenicity and possible toxicity of inorganic arsenic, but studies on toxic effects of organic arsenic and its metabolites are still limited. ${ }^{12,13}$

Recently, evidence of organic arsenical hazardous effects is slowly emerging even though the studies were limited to certain body systems. Keratosis has been observed in female workers in a chemical plant who were exposed to arsanilic acid and development of erythematous lesions on the feet and ears of rats were reported in rats exposed to dimethylarsinic acid (DMA). ${ }^{14,15}$ So far, studies on the metabolism of organic arsenicals compared to inorganic arsenic in rodents are limited. In some studies, acute exposure of organic arsenic was reported but did not result in significant accumulation of the toxicant in the liver and kidney. ${ }^{16,17}$ However, it has been observed that chronic exposure led to toxic effect in rats exposed to realgar (arsenic-containing herbal medicine) 
including decreased body weight, peripheral blood neutrality, abnormal ratio of granulocytes and lymphocytes, hypercoagulability of the blood, liver and kidney tissue damage ${ }^{18}$ as well as neurotoxicity. ${ }^{19,20}$ In a more recent and severe case, accidental ingestion of grazing pasture sprayed with MSMA had caused intense diarrhoea and dehydration in 200 cattle which subsequently led to the death of 16 animals. ${ }^{21}$

The hepatic effects of organic arsenicals on human is still insufficiently reported. However, animal studies observed that repeated exposures of monomethylarsonic acid (MMA) and DMA resulted in diffuse inflammation and hepatocellular degeneration, ${ }^{22,23}$ decrease in absolute liver weight, ${ }^{24,25}$ reduced liver glutathione, cytochrome $\mathrm{P}$ 450 content and serum ornithine decarboxylase activity. ${ }^{26,27}$ Significant neurotoxicity has also been observed in pigs exposed to oral doses of roxarsone. ${ }^{27,28}$ Even though these studies have shown that organic arsenic may have the potential deleterious effect that is yet to be discovered, evidences on the liver are still limited. ${ }^{29}$ In the present study, we examined the liver structural damage in rats chronically exposed to organic arsenic.

\section{MATERIALS AND METHODS}

\section{Ethical Approval}

The study was conducted after approval by the Institutional Animal Care and Use Committee (IIUM/ IACUC Approval/ 2016/ (9) (51)).

\section{Preparation of MSMA}

MSMA was purchased from Ancom Corp Sdn Bhd, Malaysia. The dosage given to arsenic-exposed rats was $63.20 \mathrm{mg}$ MSMA per kilogram body weight (the actual arsenic contents approximately $7.30 \mathrm{mg}$ ) to mimic arsenic exposure to humans as reported by previous study. ${ }^{29}$ The dose was also below WHO permissible limit of $10 \mu \mathrm{g} / \mathrm{mL} .{ }^{30}$ The preparation was according to Ishola et al. ${ }^{31}$

\section{Experimental animals}

Fourteen Sprague Dawley rats $(n=14)$ were randomly divided into arsenic-exposed $(n=7)$ and control $(n=7)$ groups. The animals were double-housed in polypropylene cages with wood-shaving bedding placed in animal retention area. They were acclimatized for 2 weeks and maintained under controlled condition of temperature $\left(27+2^{\circ} \mathrm{C}\right)$ and a twelve-hour light-dark cycle. After acclimatization, arsenic-exposed rats were gavaged with MSMA daily between $8 \mathrm{am}$ to $10 \mathrm{am}$. Both arsenic-exposed and control rats were allowed access to a standard rat chow (Gold Coin, Malaysia) and distilled water ad libitum. The rats were weighted daily throughout the study duration.

\section{Liver perfusion procedure}

The perfusion technique in this study was adopted from Cogger et al. ${ }^{32}$ The rats were anaesthetized with a combination of Ketamine (Ketapex, Apex Laboratories Pty Ltd., Australia), Tilatamine/ Zolazepam (Zoletil-50 VIRBAC Laboratories, Carros, France) and Xylazine (Ilium Xylazil-20, Troy Laboratories Pty Limited, Australia) based on Cheah et al. ${ }^{33}$ The animal was positioned on a board and the limbs were secured. Peritoneal cavity was carefully opened. The digestive system was placed on the side to expose the portal vein. Branula was inserted, parallel to the level of the vein to facilitate cannulation and also avoiding the risk of perforating the vessel. A low pressure flow was initiated at 3 $\mathrm{mL} / \mathrm{min}$ and the inferior vena cava was severed allowing the buffer to percolate through the liver. Then the flow was increased to $7-10 \mathrm{~mL} / \mathrm{min}$ accordingly. Once the liver was completely blanched, perfusion was continued with electron microscopy (EM) fixative until the liver hardened. The liver was harvested and the specimens were divided into 3 parts; for light microscopy, scanning and transmission electron microscopy tissue processing.

\section{Light microscopy}

Tissues for histopathological analysis were processed according to a standard procedure. Briefly, specimens were fixed in $10 \%$ neutral buffered formalin for 24 hours, dehydrated in graded concentrations of ethanol, embedded in paraffin, sectioned at $5 \mu \mathrm{m}$ in thickness and stained with haematoxylin and eosin (H\&E) for general tissue architecture, Masson's trichrome staining for connective tissue, particularly for the presence of fibrosis and Period Acid Schiff (PAS) staining for tissue glycogen content.

\section{Scanning electron microscopy (SEM)}

Liver tissue was fixed in EM fixative prepared in $0.1 \mathrm{M}$ sodium cacodylate buffer $(\mathrm{pH} 7.2)$ at $4^{\circ} \mathrm{C}$ for 24 hours. Then, it was washed with the same buffer thrice (10 minutes each) and post-fixed in 1\% osmium tetroxide at room temperature for 1 hour. Tissue block was then washed in distilled water for 2 times 
(10 minutes each). It was then dehydrated in graded seen (Figures $1 \mathrm{~A}$ and $1 \mathrm{~B}$ ). Arsenic-exposed sections concentrations of ethanol; $50 \%$ ethanol for 15 however showed hepatocytes with cytoplasmic minutes, $75 \%$ ethanol for 15 minutes, 95\% ethanol for vacuolation in zones 2 and 3 . Necrotic areas were 15 minutes twice and 100\% ethanol for 20 minutes seen with some apoptotic bodies present (Figure 1C). thrice. The tissues were then immersed in 1 to $2 \mathrm{~mL}$ Necrotic areas in Figure $1 \mathrm{C}$ were marked by the loss of hexamethyldisilazane (HMDS) for 10 minutes. HMDS of cytologic architecture of several adjacent was then decanted from the specimen vial. The hepatocytes. As for apoptosis, the hepatocytes were specimen vial with the tissues were left in the demarcated by slightly shrunken, irregularly shaped desiccator to air-dry at room temperature. The dried cells with hypereosinophilic cytoplasm and small, specimen was then mounted onto a scanning electron fragmented nuclei. There were moderate microscope (SEM) specimen stub with a double-sided inflammatory cells infiltration in the periportal areas sticky tape. Then the specimen was coated with gold consisting of lymphocytes and eosinophils (Figure (SCD Cool Sputter Coater, Bal-Tec AG) and viewed using SEM (Carl Zeiss EVO 50, Germany).

\section{Transmission electron microscopy (TEM)}

Tissue block was fixed and dehydrated in a similar manner as SEM samples except that the duration in $100 \%$ ethanol was 30 minutes. The block was then infiltrated with resin using a mixture of propylene oxide: Agar Low Viscosity resin mixed (1:1) in a rotator (Stuart SB3, Germany) for 30 minutes. Then, it was infiltrated in Agar Low Viscosity resin mixture overnight in the rotator. It was infiltrated again in a new change of Agar Low Viscosity resin mixture for about 5 hours in the rotator. Finally, the block was embedded and cured at $60^{\circ} \mathrm{C}$ for 48 hours. Block was cut to 75-85 nm thickness. Cut sections were collected on copper grids. Sections were stained with uranyl acetate and lead citrate. Sections viewed using TEM (Carl Zeiss Libra 120, Germany).

\section{RESULTS}

The mean weight of rats in control group was 470.9 (75.5) gram with an average weight increment of 6 to 10 gram per week. Arsenic-exposed rats demonstrated a lesser weight gain of about 1 to 2 gram per week with an average weight of 453.6 (73.6) gram. However, the differences in weight of both groups were not significant $(p=0.685)$.

\section{Histopathological findings}

Figure 1 shows representative of histopathological sections of control and arsenic-exposed samples. Control sample displayed normal structure of hepatocytes composed of polygonal cells arranged in plates that radiate away from central vein towards portal tracts. Regular sized sinusoids were seen in between the plates of hepatocytes. Normal hepatocytes with clearly visible nuclei were seen in all zones. No evidence of inflammation and fibrosis 1D). Moderate perisinusoidal fibrosis with focal periportal fibrosis were seen (Figure 1E) with reduced glycogen content staining (Figure 1F).
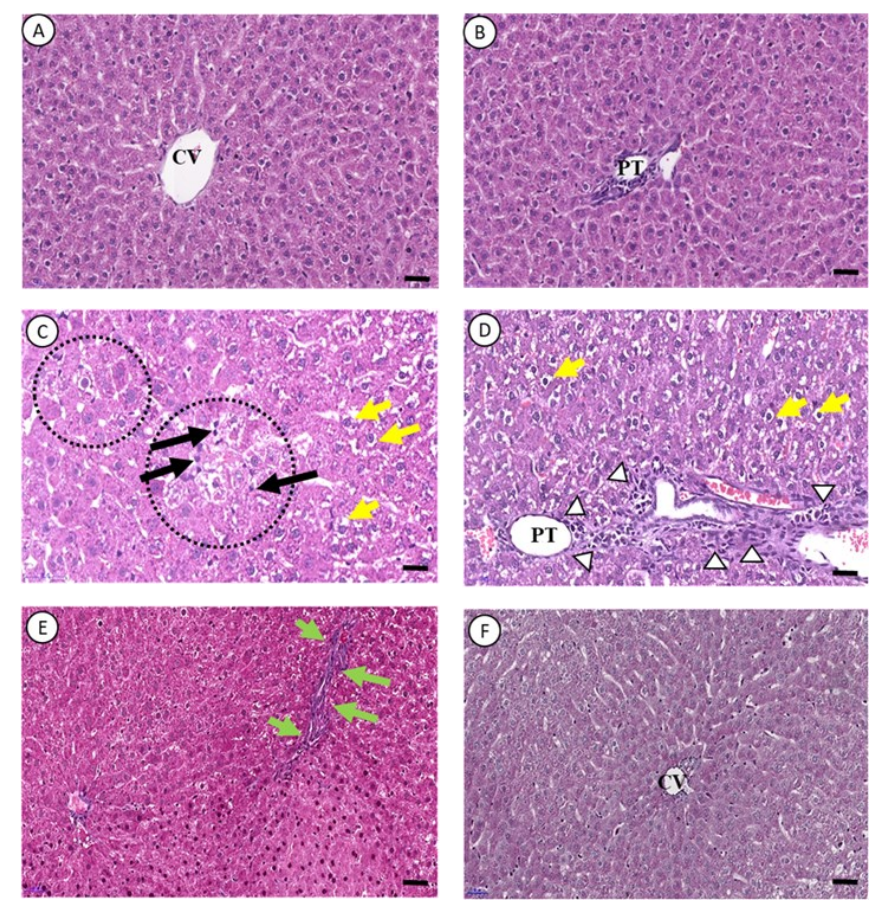

Figure 1. Representative photomicrographs of liver sections. (A) and (B): Control sample over central vein ' $\mathrm{CV}$ ' and portal area 'PT'. (C) - (F): Arsenic-exposed. (C) Areas of necrosis - dotted black circles; apoptotic bodies - black arrows; and hepatocytes cytoplasmic vacuolations - yellow arrows. (D) Inflammatory cells infiltrate - arrow heads and hepatocytes cytoplasmic vacuolations. (E) Moderate perisinusoidal fibrosis with focal periportal fibrosis - green arrows. (F) Less intense glycogen staining. (A, B, C and D: H\&E, 200X original magnification, E: Masson's trichrome staining, 100X original magnification, F: Period Acid Schiff staining, 100X original magnification. Scale bar $=50 \mu \mathrm{m}$ )

\section{Scanning electron microscopy}

Representative scanning electron micrographs for control and arsenic-exposed samples are shown in Figure 2. In control samples, hepatocytes were still preserved (Figure 2A). Sinusoids were regular (Figure 2C). Space of Disse and bile canaliculi appeared normal. Liver sinusoidal endothelial cells (LSECs) showed fenestrations (Figure 2C). In arsenic-exposed samples, disrupted hepatic cords were seen with 
numerous hepatocytes blebs (Figure 2B). Fenestrae of LSECs seemed less compared to control sample (Figure 2D).
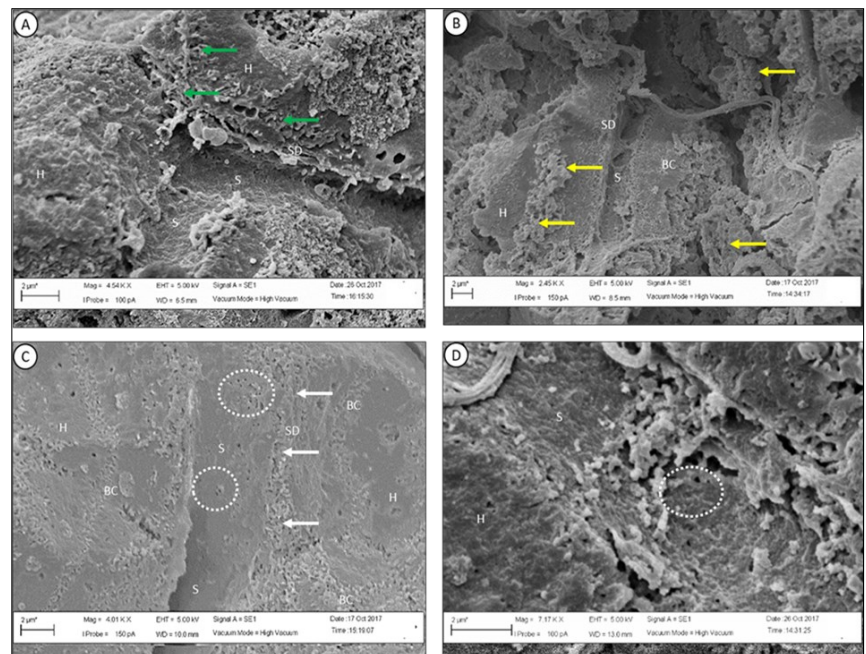

Figure 2. Representative scanning electron micrographs of rats' liver tissues. (A): Control. Normal hepatocytes ' $\mathrm{H}$ ' with regular-sized sinusoidal spaces ' $\mathrm{S}$ '. Hepatocytes microvilli (green arrows) in the space of Disse 'SD' and bile canaliculi 'BC'. (B): Arsenic-exposed group. Disrupted hepatic cords with numerous hepatocytes blebs (yellow arrows). (C): Control. Fenestrae of liver sinusoidal endothelial cells . (D): Arsenic-exposed group. Fenestrae appeared less compared to control. White-dotted circle, group of fenestrae. Magnification (A)X4500; (B)X2450, (C) X4000; (D)X7000.

\section{Transmission electron microscopy}

Transmission electron micrograph in control shows normal hepatocytes with cytoplasmic organelles (Figure 3A). In arsenic-exposed section, hepatocytes with relatively smaller nuclei were seen. Some nucleoli were not obvious. Marginalization of heterochromatin and discontinuity of nuclear membrane were observed. Cytoplasm appeared disintegrated with loss of organelles normal morphology (Figure 3B). Compared to control sample (Figure 3C), LSEC in arsenic-exposed sample showed chromatin condensation with few caveolae seen within the cytoplasm (Figure 3D).

\section{DISCUSSION}

In this study, histopathological findings showed that the effects of chronic low dose organic exposure resemble the hepatotoxicity effects of inorganic arsenic such as apoptosis, necrosis, hepatocytes with vacuolated cytoplasm, inflammatory cells infiltration and development of fibrosis. ${ }^{34}$

Several studies indicated that cytoarchitectural changes were corresponding to the increment of arsenic concentration at acute and sub chronic
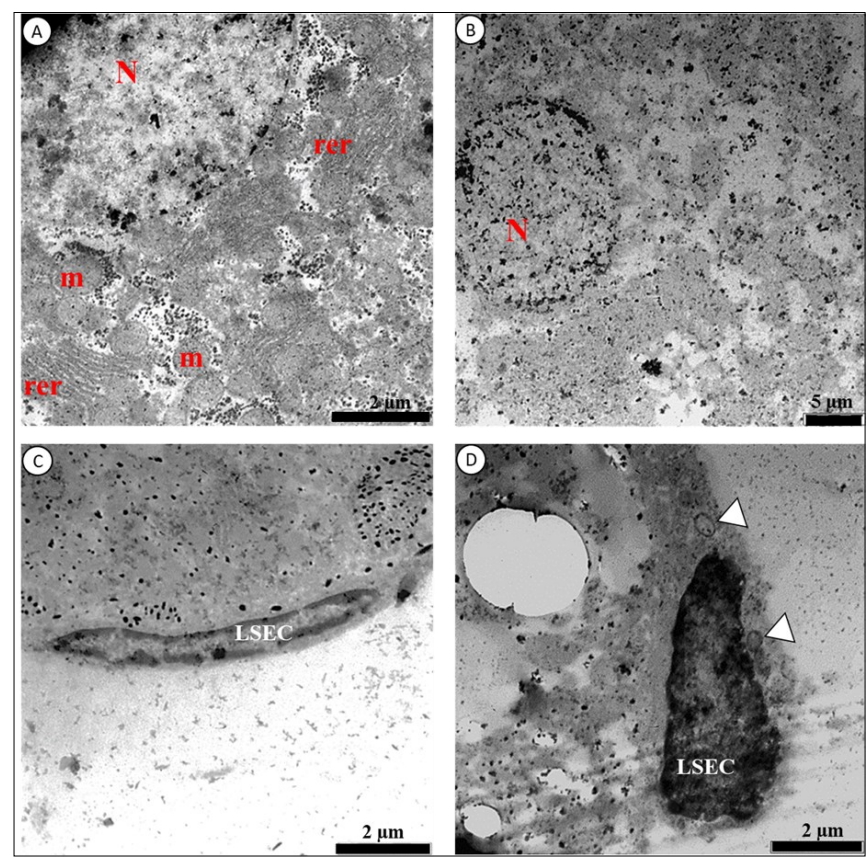

Figure 3. Representative transmission electron micrograph of rats' liver section. (A): Control. Intact hepatocyte's nucleus ' $N$ ' and normal distribution of mitochondria ' $m$ ' and rough endoplasmic reticulum 'rer'. (B): Arsenic-exposed group. Disintegrated cytoplasm and loss of organelles normal morphology. (C): Control. Normal LSEC. (D): Arsenic-exposed group. LSEC shows chromatin condensation with few caveolae (arrow heads) seen within the cytoplasm. LSEC, liver sinusoidal endothelial cell. (Original magnification, (A)X2000; (B)X 1000, (C) \& (D) X5000).

exposure including nuclear blebbing and sinusoidal spaces expansion due to necrosis of the hepatocytes..$^{35,36}$ Reduction in the number of mitochondria and rough endoplasmic reticulum were noted in chronic inorganic arsenic studies in cell culture studies; human glioblastoma cells, ${ }^{37}$ humanhamster hybrid $\mathrm{CHOK} 1$ cells $^{38}$ and mouse atrial cardiomyocytes..$^{39}$ In this study, similar observations were documented in this chronically exposed group.

Inorganic arsenic has been shown to induce mitochondrial permeability transition pore (mtPTP) opening that induces mitochondrial biochemical abnormalities as evidenced by mitochondrial swelling, mitochondrial membrane potential breakdown and membrane fluidity alterations and thus destroy inner membrane function that is important in energy production. ${ }^{40}$ Arsenic trioxide triggered endoplasmic reticulum (ER) stress in different types of non-malignant cells such as myoblasts, vascular endothelial cells, pancreatic bcells, neutrophils, and macrophages. ${ }^{41,42}$ ER stress could lead to several deleterious effects within cells and tissues including accumulation of lipids, cell death, cytolysis, and inflammation. ${ }^{43}$ 
Recent study highlighted that ER and mitochondria work in tandem especially at the site where they are in close contact. Contact sites are defined as regions where two membranes are closely apposed but the membranes do not fuse and thus the organelles still maintain their identities. ${ }^{44}$ This contact has been currently recognized plays an emerging role in coordinating the functions between ER and mitochondria in regulating lipid synthesis, calcium signalling and controlling mitochondrial biogenesis and intracellular trafficking. ${ }^{28,45}$ Therefore, defects in these ER-mitochondria junction (or relationship) will affect cell dynamic and lead to cell dysfunction. In this study, MSMA may have as well induced ER stress which possibly explains the loss of organelles normal morphology in the hepatocytes. The loss of organelles normal morphology was based on comparison with several photomicrographs of control rats, viewed at random. It is probable that the reduction in the number of ER and mitochondria may have occurred together with the loss of organelle morphology.

Apoptosis is characterized by cellular and nuclear shrinkage as well as budding or blebbing which lead to pinching off blebs giving rise to apoptotic bodies and chromatin condensation. ${ }^{46}$ Blebs formation appears to be a prominent feature of most forms of liver injury regardless of origin. ${ }^{47}$ This structure was prominent in the arsenic-exposed rats in this study.

Capillarization is the angiogenic process of the liver sinusoidal endothelium that results in ultrastructural phenotypic conversion marked by loss of fenestration and tight intercellular junction. This event is shown fundamental to the pathogenesis of a number of diseases caused by environmental arsenic exposures, including cardiovascular ischemic diseases, atherosclerosis, tumorigenesis, and liver fibrosis. ${ }^{48,49}$ In an animal study, Straub et al. had demonstrated that acute low dose inorganic exposure decreased fenestral size and overall sinusoid porosity even though at that exposure hepatocytes were preserved..$^{50}$ Our study findings observed a comparatively lesser fenestrae as opposed to control rats. Even though quantitation of the fenestrae would provide an objective comparison between the two groups which however were not able to be performed in the study, arsenic-exposed rats did not display regular arrangements of fenestral groups as observed in control rats. Thus, MSMA might also have the capability to induce capillarization in the LSEC.

\section{CONCLUSION}

Chronic Monosodium methylarsonate (MSMA) exposure results in necrotic and apoptotic changes of the liver with the loss of organelles normal morphology in the hepatocytes and defenestration of liver sinusoidal endothelial cells (LSEC). This study shows chronic low dose organic arsenic exposure is toxic to hepatocytes. Nevertheless, LSEC demonstrated capillarization changes.

\section{AUTHOR CONTRIBUTIONS}

All authors contributed toward data analysis, drafting and critically revising the paper and agree to be accountable for all aspects of the work.

\section{DISCLOSURE OF CONFLICT OF INTEREST}

The authors have no disclosures to declare.

\section{COMPLIANCE WITH ETHICAL STANDARDS}

The work is compliant with ethical standards.

\section{ACKNOWLEDGEMENTS}

This research was supported by the grant from Ministry of Higher Education, Malaysia (FRGS 15-2160457).

\section{REFERENCES}

1. Kim T, Seo J, Hong Y, Song K. Case-control study of chronic low-level exposure of inorganic arsenic species and non-melanoma skin cancer. J Dermatol. 2017;44(12):1374-9.

2. Goessler W, Brauer S, Milić M, Šerić V, Milić M, et al. Measurement of arsenic, arsenic species and other elements in urine, drinking water and hair samples-screening of the situation in Eastern Croatia. In: 10th Congress of Toxicology in Developing Countries (IUTOX CTDC10) and 12th Congress of the Serbian Society of Toxicology (12th SCT). 2018.

3. Aposhian H, Gurzau E, Gurzau A, Healy S, Lu X, et al. Occurrence of monomethylarsonous acid in urine of humans exposed to inorganic arsenic. Chem Res Toxicol. 2000;13:693-7.

4. Vergara PM, Soto GE, Moreira-Arce D, Rodewald $A D$, Meneses LO, et al. Foraging behaviour in magellanic woodpeckers is consistent with a multi-scale assessment of tree quality. PLoS One. 2016;11(7):e0159096.

5. Petursdottir AH, Sloth JJ, Feldmann J. Introduction of regulations for arsenic in feed 
and food with emphasis on inorganic arsenic, and implications for analytical chemistry. Anal Bioanal Chem. 2015;407(28):8385-96.

6. Hammid ANA, Kuntom A, Ismail R, Pardi N. Determination of arsenic in palm kernel expeller using microwave digestion and graphite furnace atomic absorption spectrometry method. Int Journl Basicand. 2013;1(3):641-649

7. Poprac P, Jomova K, Simunkova M, Kollar V, Rhodes CJ, et al. Targeting free radicals in oxidative stress-related human diseases. Trends Pharmacol Sci. 2017;38(7):592-607.

8. Valko M, Rhodes C, Moncol J, Izakovic M, Mazur $M$. Free radicals, metals and antioxidants in oxidative stress-induced cancer. Chem Biol Interact. 2006;160:1-40.

9. Aposhian H, Zheng B, Aposhian M, Le X, Cebrian $M$, et al. DMPS-arsenic challenge test. II. Modulation of arsenic species, including monomethylarsonous acid (MMA(III)), excreted in human urine. Toxicol Appl Pharmacol. 2000;165:74-83.

10. Kobayashi $Y$, Hirano S. Distribution and excretion of arsenic metabolites after oral administration of seafood-related organoarsenicals in rats. Metals (Basel). 2016;6 (10):231.

11. Mohammed Abdul KS, Jayasinghe SS, Chandana EPS, Jayasumana C, De Silva PMCS. Arsenic and human health effects: A review. Environ Toxicol Pharmacol [Internet]. 2015;40(3):828-46. Available from: http://dx.doi.org/10.1016/ j.etap.2015.09.016

12. Sarkar A, Paul B. The global menace of arsenic and its conventional remediation-A critical review. Chemosphere. 2016;158:37-49.

13. Singh R, Singh S, Parihar P, Singh VP, Prasad SM. Arsenic contamination, consequences and remediation techniques: a review. Ecotoxicol Environ Saf. 2015;112:247-70.

14. Chou S, Harper C, ATSDR, Division of Toxicology and Environmental Medicine, Atlanta G, Ingerman L, Llados F, Colman J, et al. Chemical and Physical Information [Internet]. Vol. 16, Toxicology and Industrial Health. 2007. p. 87-92. Available from: https://www.atsdr.cdc.gov/ toxprofiledocs/index.html

15. Gupta PK. Toxic effects of metals and micronutrients. In: Concepts and Applications in Veterinary Toxicology. Springer; 2019. 83-119.

16. Lewchalermvong K, Rangkadilok N, Nookabkaew S, Suriyo T, Satayavivad J. Arsenic speciation and accumulation in selected organs after oral administration of rice extracts in Wistar rats. $\mathrm{J}$ Agric Food Chem [Internet]. 2018 Mar 28 [cited 2019 Jan 16];66(12):3199-209.

17. Mandal P. An insight of environmental contamination of arsenic on animal health. Emerg Contam. 2017;3(1):17-22.

18. Yi Y, Gao S, Xia J, Zhao Y, Li C, et al. Dimethylarsenic acid (DMA) accumulation positively correlates with realgar-induced subchronic toxicity in rats. bioRxiv. 2018;398206.

19. Huo T, Li W, Zhang Y, Yuan J, Gao L, et al. Excitotoxicity induced by realgar in the rat hippocampus: the involvement of learning memory injury, dysfunction of glutamate metabolism and NMDA receptors. Mol Neurobiol. 2015;51(3):980-94.

20. Saha S, Sadhukhan P, Mahalanobish S, Dutta S, Sil PC. Ameliorative role of genistein against age -dependent chronic arsenic toxicity in murine brains via the regulation of oxidative stress and inflammatory signaling cascades. J Nutr Biochem [Internet]. 2018 [cited 2019 Jan 16];55:26-40.

21. Gonçalves MA, da Cruz RAS, Reis MO, Pavarini SP, Sonne L, Driemeier D. Vascular injury in spontaneous subacute toxicosis caused by organic arsenic in cattle. Pesqui Vet Bras. 2017;37(7):676-80.

22. Masuda T, Ishii K, Morishita Y, Iwasaki N, Shibata Y, Tamaoka A. Hepatic histopathological changes and dysfunction in primates following exposure to organic arsenic diphenylarsinic acid. J Toxicol Sci [Internet]. 2018 [cited 2019 Apr 19];43(5):291-8.

23. Siewicki T. Tissue retention of arsenic in rats fed witch flounder or cacodylic acid. J Nutr. 1981;111:602-9.

24. Shimoda Y, Kato K, Asami S, Kurita M, Kurosawa $\mathrm{H}$, Toriyama $M$, et al. Differences in apoptotic signaling and toxicity between dimethylmonothioarsinic acid (DMMTAV) and its active metabolite, dimethylarsinous acid (DMAIII), in HepaRG cells: Possibility of apoptosis cascade based on diversity of active metabolites of DMMTAV. J Trace Elem Med Biol. 2018;50:188-97.

25. Ahmad S, Anderson W, Kichin K. Dimethylarsinic acid effects on DNA damage and oxidative stress related biochemical parameters in B6C3F1 mice. Cancer Lett. 1999;139:129-35. 
26. Ohfuji S. Spinal cord degeneration in juvenile and adult swine. Comp Clin Path. 2018;27 (5):1375-81.

27. Kennedy S, Rice D, Cush P. Neuropathology of experimental 3-nitro-4-hydroxyphenylarsonic acid toxicosis in pigs. Vet Pathol. 1986;23:45461.

28. Prakash C, Soni M, Kumar V. Mitochondrial oxidative stress and dysfunction in arsenic neurotoxicity: A review [Internet]. Vol. 36, Journal of Applied Toxicology. 2016 [cited 2019 Jan 16]. p. 179-88.

29. Mandal P. An insight of environmental contamination of arsenic on animal health. Emerg Contam [Internet]. 2017 Mar 1 [cited 2019 Jan 16];3(1):17-22.

30. WHO. Guidelines for Drinking-Water Quality, 4th ed. World Health Organization, Geneva [Internet]. World Health Organization. 2017 [cited 2018 Oct 3]. p. 631. Available from: https://www.who.int/water_ sanitation_health/publications/drinking-waterquality-guidelines-4-including-1st-addendum/ en/

31. Ishola AA, A.Talib N, Muhammad N, Buyong Z, Mohamed AH, et al. Organic arsenical exposure stimulates atherosclerosis through oxidative stress increase and adhesion molecule expression. J Appl Pharm Sci. 2016;6(11):40-51.

32. Cogger VC, O'Reilly JN, Warren A, Le Couteur DG. A standardized method for the analysis of liver sinusoidal endothelial cells and their fenestrations by scanning electron microscopy. J Vis Exp [Internet]. 2015;(98):1-9.

33. Cheah PS, Low KC, Mohidin N, Bariah MA, Maung $M$, et al. Ketamine-xylazine/ tiletaminezolazepam: prolonged anesthesia in cynomolgus monkeys. Online J Vet Res. 2007;11:39-45.

34. Maji C, Sarkar S, Biswas S, Patra PH, Datta BK, et al. Experimental assessment of arsenic toxicity in garole sheep in India. Emerg Contam [Internet]. 2016 Sep 1 [cited 2019 Jan 16];2 (3):128-34.

35. Gaim K, Gebru G, Abba S. The effect of arsenic on liver tissue of experimental animals (fishes and mice)-a review article. Int J Sci Res Publ. 2015;5(5):1-9.

36. Al-Forkan $M$, Islam $S$, Akter R, Shameen Alam S, Khaleda $L$, et al. A sub-chronic exposure study of arsenic on hematological parameters, liver enzyme activities, histological studies and accumulation pattern of arsenic in organs of wistar albino rats. J Cytol Histol S5(06):S5-006

37. Haga N, Fujita N, Tsuruo T. Involvement of mitochondrial aggregation in arsenic trioxide (As203)- induced apoptosis in human glioblastoma cells. Cancer Sci. 2005;96(11):82533.

38. Partridge $M$, Huang $S$, Hernandez-Rosa E, Davidson M, Hei T. Arsenic induced mitochondrial DNA damage and altered mitochondrial oxidative function: Implications for genotoxic mechanisms in mammalian cells. Cancer Res. 2007;67(11):5239-47.

39. Watanabe R, Unuma K, Noritake K, Funakoshi $T$, Aki $T$,et al. Ataxia telangiectasia and rad3 related (ATR)-promyelocytic leukemia protein (PML) pathway of the DNA damage response in the brain of rats administered arsenic trioxide. J Toxicol Pathol [Internet]. 2017;30(4):333-7.

40. Jiao YH, Zhang Q, Pan LL, Chen XY, Lei KL, Zhao $\mathrm{J}$, et al. Rat Liver Mitochondrial Dysfunction Induced by an Organic Arsenical Compound 4-(2Nitrobenzaliminyl) Phenyl Arsenoxide. J Membr Biol. 2015;248(6):1071-8.

41. King Y, Chiu Y, Chen H, Kuo K, Lu C, et al. Endoplasmic reticulum stress contributes to arsenic trioxide-induced intrinsic apoptosis in human umbilical and bone marrow mesenchymal stem cells. Env Toxicol. 2015;

42. Weng $C$, Chiou S, Wang L, Kou M, Wang $Y$, et al. Arsenic trioxide induces unfolded protein response in vascular endothelial cells. Arch Toxicol. 2014;88:213-26.

43. Foufelle F, Fromenty B. Role of endoplasmic reticulum stress in drug-induced toxicity. Pharmacol Res Perspect. 2016;4(1):1-28.

44. Rowland AA, Voeltz GK. Endoplasmic reticulummitochondria contacts: Function of the junction. Nat Rev Mol Cell Biol. 2016;13(10):607 $-25$.

45. Pace C, Banerjee TD, Welch B, Khalili R. Dagda RK, et al. Monomethylarsonous acid, but not inorganic arsenic, is a mitochondria- specific toxicant in vascular smooth muscle cells. 2015;91(2):165-71.

46. Liu F, Dai S, Feng D, Peng X, Qin Z, et al. Versatile cell ablation tools and their applications to study loss of cell functions. Cell Mol Life Sci. 2019;1-19.

47. Walker AM, Stevens JJ, Ndebele K, Tchounwou PB. Evaluation of arsenic trioxide potential for lung cancer treatment: assessment of apoptotic 
mechanisms and oxidative damage. J Cancer Sci Ther. 2016;08(01):1-9.

48. DeLeve L, Wang X, Hu L, McCuskey M, McCuskey R. Rat liver sinusoidal endothelial cell phenotype is maintained by paracrine and autocrine regulation. Am J Physiol Gastrointest Liver Physiol. 2004;287:G757-63.

49. Straub AC, Stolz DB, Vin $H$, Ross MA, Soucy $N$ V., Klei LR, et al. Low level arsenic promotes progressive inflammatory angiogenesis and liver blood vessel remodeling in mice. Toxicol Appl Pharmacol. 2007;222(3):327-36.

50. Straub AC, Stolz DB, Ross MA, Hernández-Zavala A, Soucy NV., et al. Arsenic stimulates sinusoidal endothelial cell capillarization and vessel remodeling in mouse liver. Hepatology. 2007;45(1):205-12. 